Pacific Journal of Mathematics

HOMOMORPHISMS OF GROUP ALGEBRAS WITH NORM 


\title{
HOMOMORPHISMS OF GROUP ALGEBRAS
}

\section{WITH NORM LESS THAN $\sqrt{2}$}

\author{
N. J. Kalton And G. V. Wood
}

We show that two locally compact abelian groups $G_{1}$ and $G_{2}$ are isomorphic if there exists an algebra isomorphism $T$ of $L^{1}\left(G_{1}\right)$ onto $L^{1}\left(G_{2}\right)$ with $\|T\|<\sqrt{2}$. This constant is best possible. The same result is proved for locally compact connected groups, but for the general locally compact group, the result is proved under the hypothesis $\|T\|<1.246$. Similar results are given for the algebras $C(G)$ and $L^{\infty}(G)$ when $G$ is compact. In the abelian case, we give a representation theorem for isomorphisms satisfying $\|T\|<\sqrt{2}$.

1. Introduction. In [13], Wendel proved that, for locally compact groups $G_{1}$ and $G_{2}$, if $T$ is an algebra isomorphism of $L^{1}\left(G_{1}\right)$ onto $L^{1}\left(G_{2}\right)$ with $\|T\| \leqq 1$, then $G_{1}$ and $G_{2}$ are isomorphic. Similar results for $M(G), C_{c}(G)$ and $L^{p}(G)$ have been proved in [3], [5], [6], [7], [11], [12], [14] and [15]. For abelian groups, better results are known. In [8], it is shown that two locally compact abelian groups $G_{1}$ and $G_{2}$ are isomorphic if there exists an algebra isomorphism $T$ of $L^{1}\left(G_{1}\right)$ onto $L^{1}\left(G_{2}\right)$ with $\|T\|<\frac{1}{2} \sqrt{5}$, and in view of a result of Saeki in [9], this can be improved to the condition $\|T\|<\frac{1}{2}(1+\sqrt{2}$ ), (see [8] 4.6.3 (c)). Saeki's later paper [10] makes it possible to extend the result even further to the condition $\|T\|<\frac{1}{4}(1+\sqrt{17})$.

We prove the result for abelian groups with the condition $\|T\|<\sqrt{2}$ and this is the best possible constant, as Example 1 shows. In fact we characterize all algebra isomorphisms $T$ of $L^{1}\left(G_{1}\right)$ onto $L^{1}\left(G_{2}\right)$ with $\|T\|<\sqrt{2}$ as follows:

Case 1. If $0<\|T\|<\frac{1}{2}(1+\sqrt{ } 3)$, then $T$ has the form

$$
(T f)(x)=\psi(x) f(s(x))
$$

where $s: G_{2} \rightarrow G_{1}$ is a topological isomorphism and $\psi \in \hat{G}_{2}$. In this case $T$ is an isometry.

Case 2. If $\frac{1}{2}(1+\sqrt{3}) \leqq\|T\|<\sqrt{2}$, then $T$ has the form

$$
(T f)(x)=\psi(x)\left\{\frac{1}{2}(1+\varphi(x)) f(s(x))+\frac{1}{2}(1-\varphi(x)) f(s(x) u)\right\}
$$


where $s: G_{2} \rightarrow G_{1}$ is a topological isomorphism, $\psi, \varphi \in \hat{G}_{2}$ with $\varphi$ of odd order $n$, and $u \in G_{1}$ is an element of order 2. In this case $\|T\|=$ $\sqrt{2} \cos \pi / 4 n$.

Again $\frac{1}{2}(1+\sqrt{3})$ is the best possible constant as Example 2 shows. It is interesting to note that the isomorphisms given in case (2) above correspond to the idempotents characterized by Saeki in [10]. We give similar results for homomorphisms. In [8] Chapter 4, algebra homomorphisms of $L^{1}\left(G_{1}\right)$ into $M\left(G_{2}\right)$ are represented by means of piecewise affine maps from a subset of $\hat{G}_{2}$ to $\hat{G}_{1}$, (see [8] p. 78 for definitions). Our proofs do not however use this representation. We prove the result first for discrete groups by a computational argument, and then use this to prove the general result. An advantage of our method is that it generalises to give corresponding results in the nonabelian case. We show that if $G_{1}$ and $G_{2}$ are locally compact connected groups and $T$ is an algebra isomorphism of $L^{1}\left(G_{1}\right)$ onto $L^{1}\left(G_{2}\right)$ with $\|T\|<\sqrt{2}$, then $G_{1}$ and $G_{2}$ are isomorphic. Without connectedness, we can prove the result under the condition $\|T\|<\lambda_{0}$ where $\lambda_{0}$ is the root of a cubic equation $\left(\lambda_{0} \sim 1 \cdot 247\right)$. In these cases we cannot describe the form of the isomorphism, nor do we know whether these constants are best possible. There are corresponding results in each case for isomorphisms of the convolution algebras $M(G), L^{\infty}(G)$ and $C(G)$ (in the latter cases we assume $G$ compact).

The idea for the paper came from the generalization of the BanachStone theorem due to Cambern [2] and Amir [1] which states that, for compact Hausdorff spaces $X$ and $Y$, if $T: C(X) \rightarrow C(Y)$ is a linear isomorphism with $\|T\|\left\|T^{-1}\right\|<2$, then $X$ and $Y$ are homeomorphic. In the event, we were able to bypass this result because the extra condition we have when $X$ and $Y$ are groups, that $T$ is a convolution algebra isomorphism enables us to find the map from $X$ to $Y$ directly and under the weaker hypothesis of $\|T\|<\sqrt{2}$.

Notation. As usual, $C(G), L^{1}(G), L^{\infty}(G)$ and $M(G)$ will denote the continuous functions, the integrable functions, the bounded measurable functions, and the bounded measures respectively on a locally compact group $G$. To avoid confusion we will write $l_{1}(G)$ in place of $L^{1}(G)$ when $G$ is discrete. We will use $f, g$, etc. to denote elements of $l_{1}(G)$ or $L^{1}(G)$ and $x, y, z$, u, etc. to denote elements of the group $G$ or the corresponding elements of $l_{1}(G)$. $\quad \hat{G}$ will denote the dual group of $G$ if $G$ is abelian, and $\hat{f}$ the Fourier transform of $f \in L^{1}(G)$. If $s$ is a homomorphism of $G_{1}$ into $G_{2}$, then $\hat{s}$ will denote the induced homomorphism of $\hat{G}_{2}$ into $\hat{G}_{1}$.

We begin with some examples from finite groups. Let $\mathbf{Z}_{n}$ denote the cyclic group of order $n$. 
EXAMPLE 1. The group algebras of $\mathbf{Z}_{4}$ and $\mathbf{Z}_{2} \times \mathbf{Z}_{2}$ are isomorphic under the map defined by

$$
T x=\frac{1}{2}(1+i) y+\frac{1}{2}(1-i) z
$$

where $x$ is a generator of $\mathbf{Z}_{4}$ and $y, z$ are generators of $\mathbf{Z}_{2} \times \mathbf{Z}_{2}$. As a map of $l_{1}\left(\mathbf{Z}_{4}\right)\left(=L^{1}\left(\mathbf{Z}_{4}\right)\right)$ into $l_{1}\left(\mathbf{Z}_{2} \times \mathbf{Z}_{2}\right)$ (or $C\left(\mathbf{Z}_{4}\right)$ into $C\left(\mathbf{Z}_{2} \times \mathbf{Z}_{2}\right)$ ) $\|T\|=\sqrt{2}$.

EXAMPLE 2. There is an isomorphism of $l_{1}\left(\mathbf{Z}_{6}\right)\left(\right.$ or $\left.C\left(\mathbf{Z}_{6}\right)\right)$ into itself which is not induced directly by a group isomorphism. If $x$ is a generator of $\mathbf{Z}_{6}$ define $T$ by

$$
T x=-\frac{1}{2} x+\frac{i \sqrt{3}}{2} x^{4}
$$

Then $\|T\|=\frac{1}{2}(1+\sqrt{ } 3)$ which is less than $\sqrt{ } 2$.

EXAMPLE 3. There is an algebra embedding of $l_{1}\left(\mathbf{Z}_{2}\right)$ in $l_{1}\left(\mathbf{Z}_{4}\right)$ of small norm which is not induced by a group map. If $x$ generates $\mathbf{Z}_{2}$ and $y$ generates $\mathbf{Z}_{4}$, define $T$ by

$$
T x=\frac{1}{4}(1-i) y+\frac{1}{2} y^{2}+\frac{1}{4}(1+i) y^{3} .
$$

Then $T x^{2}=\frac{1}{2} e+\frac{1}{4}(1+i) y+\frac{1}{4}(1-i) y^{3}$ which is an idempotent in $l_{1}\left(\mathbf{Z}_{4}\right)$. Also $\|T\|=\frac{1}{2}(1+\sqrt{2})$.

EXAMPLE 4. There exists an algebra epimorphism of $l_{1}\left(\mathbf{Z}_{6}\right)$ onto $l_{1}\left(\mathbf{Z}_{2} \times \mathbf{Z}_{2}\right)$ which is induced by a group homomorphism which is not an epimorphism. Let $x$ generate $\mathbf{Z}_{6}, y$ and $z$ generate $\mathbf{Z}_{2} \times \mathbf{Z}_{2}$, and define $T$ by

$$
T x=\frac{1}{2} y-\frac{i \sqrt{3}}{2} z
$$

Then $T x^{2}=-\frac{1}{2} e-(i \sqrt{3} / 2) y z$ and $T x^{3}=-y$, and $T$ is clearly an epimorphism, but there is no epimorphism of $\mathbf{Z}_{6}$ onto $\mathbf{Z}_{2} \times \mathbf{Z}_{2}$. In this case $\|T\|=\frac{1}{2}(1+\sqrt{3})$.

The dual map $T^{*}$ is a monomorphism of $C\left(\mathbf{Z}_{2} \times \mathbf{Z}_{2}\right)$ into $C\left(\mathbf{Z}_{6}\right)$ which is not induced by an epimorphism, (see Theorem 3.5).

2. Discrete abelian groups. In this section we shall study algebra homomorphisms $T: l_{1}\left(G_{1}\right) \rightarrow l_{1}\left(G_{2}\right)$ which preserve the 
identity. Although we shall restrict attention to the abelian case, our first result is true for any pair of groups $G_{1}$ and $G_{2}$, and we shall use it later in $\S 4$. We shall denote the identities of $G_{1}$ and $G_{2}$ by $e_{1}$ and $e_{2}$ respectively.

Proposition 2.1. Let $G_{1}$ and $G_{2}$ be any two groups and suppose $T: l_{1}\left(G_{1}\right) \rightarrow l_{1}\left(G_{2}\right)$ is a bounded algebra homomorphism such that $T e_{1}=$ $e_{2}$. Then if $x \in G_{1}$ and $T x=\sum_{t=1}^{\infty} \alpha_{1} y_{t}$ where $y_{i} \in G_{2}$ are distinct, then there exists $j$, such that $\left|\alpha_{\jmath}\right| \geqq\|T\|^{-1}$. If $\|T\|<\sqrt{2}$, then $j$ is unique.

Proof. Let $T x^{-1}=\sum_{t=1}^{\infty} \beta_{i} y_{l}^{-1}+\sum_{l=1}^{\infty} \gamma_{t} z_{l}$ where the $\left(z_{i}\right)$ are distinct and disjoint from $\left(y_{1}^{-1}\right)$. Then $e_{2}=T x * T x^{-1}$ and so identifying the coefficient of $e_{2}$ we obtain $\sum_{l=1}^{\infty} \alpha_{\imath} \beta_{l}=1$. Hence

$$
\begin{aligned}
1 & \leqq \sup \left|\alpha_{\imath}\right|\left(\Sigma\left|\beta_{i}\right|\right) \\
& \leqq\left(\sup \left|\alpha_{\imath}\right|\right)\left\|T x^{-1}\right\|
\end{aligned}
$$

so that $\sup \left|\alpha_{\imath}\right| \geqq\|T\|^{-1}$. Since $\alpha_{\imath} \rightarrow 0$, there exists $j$ such that $\left|\alpha_{l}\right| \geqq$ $\|T\|^{-1}$.

Suppose $\|T\|<\sqrt{2}$, and $k$ is another index such that $\left|\alpha_{k}\right| \geqq$ $\|T\|^{-1}$. Then $\|T x\| \geqq\left|\alpha_{j}\right|+\left|\alpha_{k}\right| \geqq 2 \cdot\|T\|^{-1} \geqq \sqrt{2}$, which is a contradiction.

On $l_{1}(G)$, there is an involution defined by $\left(\sum \alpha_{t} x_{t}\right)^{*}=\sum \bar{\alpha}_{t} x_{t}^{-1}$. We shall also define the $l_{2}$-norm by $\left\|\Sigma \alpha_{\imath} x_{t}\right\|_{2}=\sqrt{\left(\Sigma\left|\alpha_{i}\right|^{2}\right.}$ (where the $\left(x_{t}\right)$ are distinct).

Proposition 2.2. Suppose $G_{1}$ and $G_{2}$ are abelian groups and $T: l_{1}\left(G_{1}\right) \rightarrow l_{1}\left(G_{2}\right)$ is a bounded algebra homomorphism such that $T e_{1}=$ $e_{2}$. Then $T$ is $a^{*}{ }^{*}$ map and for $x \in G_{1},\|T x\|_{2}=1$.

Proof. Let $\chi$ be any character on $G_{2}$. Then $\chi$ induces a multiplicative linear functional $\tilde{\chi}: l_{1}\left(G_{2}\right) \rightarrow C$. $\tilde{\chi} \circ T$ is multiplicative on $l_{1}\left(G_{1}\right)$, and hence for $f \in l_{1}\left(G_{1}\right)$

$$
\tilde{\chi}\left(T f^{*}\right)=\overline{\tilde{\chi}(T f)}
$$

This is true for any $\chi \in \hat{G}_{2}$ and hence $T f^{*}=(T f)^{*}$ (since $l_{1}\left(G_{2}\right)$ is semi-simple).

Now $e_{2}=T x * T x^{-1}=T x *(T x)^{*}$. Hence if $T x=\sum_{i=1}^{\infty} \alpha_{l} y_{t}$, we have, identifying the coefficient of $e_{2}$

$$
1=\Sigma\left|\alpha_{1}\right|^{2}=\|T x\|_{2}^{2} \text {. }
$$


For the next three lemmas, we assume that $G_{1}$ and $G_{2}$ are abelian and $T: l_{1}\left(G_{1}\right) \rightarrow l_{1}\left(G_{2}\right)$ is an algebra homomorphism such that $T e_{1}=e_{2}$ and $\|T\|<\sqrt{2}$. We define, for $x \in G_{1}, t(x) \in G_{2}$ by

$$
T x=\alpha t(x)+\sum_{i=1}^{\infty} \beta_{\imath} y_{\imath}
$$

where $|\alpha| \geqq\|T\|^{-1}$ and $t(x) \neq y_{i}$ for any $i . \quad t$ is then a well-defined map by Proposition 2.1. It follows from the fact that $T$ is a ${ }^{*}$-map that $t\left(x^{-1}\right)=(t(x))^{-1}$. However, in general, $t$ is not a homomorphism. The next two lemmas investigate the consequences of $t\left(x^{2}\right)$ being equal or not equal to $(t(x))^{2}$.

Lemma 2.3. Let $x \in G_{1}$ and $t\left(x^{2}\right)=(t(x))^{2}$. Suppose $T x=$ $\alpha t(x)+f$ and $T x^{2}=\beta t\left(x^{2}\right)+g$ where $f$ and $g$ contain no terms in $t(x)$ and $t\left(x^{2}\right)$ respectively. Then $\|g\| \geqq\left(2 /\|T\|^{2}\right)\|f\|$.

Proof. By multiplication

$$
T x^{2}=\alpha^{2} t(x)^{2}+2 \alpha t(x) * f+f * f .
$$

We consider two cases:

(a) If

$$
|\alpha| \geqq\|T\|^{-\frac{1}{2}}
$$

then

$$
\begin{aligned}
\|g\| & \geqq 2|\alpha|\|f\|-\|f\|^{2} \\
& =(3|\alpha|-\|T x\|)\|f\| \\
& \geqq\left(3\|T\|^{-\frac{1}{2}}-\|T\|\right)\|f\| .
\end{aligned}
$$

For $1 \leqq \theta \leqq 2^{\frac{3}{4}}$, let $\varphi(\theta)=3 \theta-\theta^{2}$, then since $\varphi^{\prime \prime}(\theta)=-2, \varphi$ is concave and so

$$
\varphi(\theta) \geqq \min \left(\varphi(1), \varphi\left(2^{\frac{3}{4}}\right)\right) \text { for } \quad 1 \leqq \theta \leqq 2^{\frac{3}{4}} \text {. }
$$

Hence

$$
3 \theta-\theta^{2} \geqq 2
$$

(since $\varphi(1)=2$, and $\left.\varphi\left(2^{\frac{3}{4}}\right)=2\left(3.2^{-\frac{1}{4}}-\sqrt{ } 2\right)>2\right)$. Thus since $1 \leqq\|T\| \leqq$ $\sqrt{ } 2$, and letting $\theta=\|T\|^{\frac{3}{2}}$

$$
\|g\| \geqq \frac{2}{\|T\|^{2}}\|f\| .
$$

(b) Next suppose $|\alpha|<\|T\|^{-\frac{1}{2}}$ (note that this implies that $\|T\|>$ 1). For convenience we shall let $k=\|T\|^{-\frac{1}{2}}$. Then $f * f$ will contain a term in $t(x)^{2}$ of magnitude at least $|\beta|-|\alpha|^{2} \geqq k^{2}-|\alpha|^{2}>0$. Hence 


$$
\begin{aligned}
\|g\| & \geqq 2|\alpha|\|f\|-\|f\|^{2}+k^{2}-|\alpha|^{2} \\
& =k^{2}-(|\alpha|-\|f\|)^{2}
\end{aligned}
$$

and, as $\|f\| \neq 0,\|g\| /\|f\| \geqq 1 /\|f\|\left(k^{2}-(|\alpha|-\|f\|)^{2}\right)$. If we define

then

$$
\varphi(\theta)=\theta^{-1}\left(k^{2}-(|\alpha|-\theta)^{2}\right)
$$

$$
\varphi^{\prime}(\theta)=\theta^{-2}\left(|\alpha|^{2}-k^{2}\right)-1 \leqq 0 \text { for } \quad \theta>0 .
$$

Hence as $\|f\| \leqq\|T\|-|\alpha|$

$$
\frac{\|g\|}{\|f\|} \geqq \frac{1}{k^{-2}-|\alpha|}\left(k^{2}-\left(2|\alpha|-k^{-2}\right)^{2}\right),
$$

where $k^{2}<|\alpha|<k$. If we write $\gamma=k^{-2}-|\alpha|$

$$
\frac{\|g\|}{\|f\|} \geqq \frac{1}{\gamma}\left(k^{2}-\left(k^{-2}-2 \gamma\right)^{2}\right)
$$

and $k^{-2}-k<\gamma<k^{-2}-k^{2}$.

If

$$
\psi(\theta)=\theta^{-1}\left(k^{2}-\left(k^{-2}-2 \theta\right)^{2}\right)
$$

then

$$
\psi^{\prime}(\theta)=\theta^{-2}\left(k^{-4}-k^{2}\right)-4
$$

and so $\psi^{\prime \prime}(\theta) \leqq 0$ for $\theta \geqq 0$. Therefore $\psi$ is concave and so for $k^{-2}-k<$ $\gamma<k^{-2}-k^{2}$

$$
\psi(\gamma) \geqq \min \left(\psi\left(k^{-2}-k\right), \psi\left(k^{-2}-k^{2}\right)\right)
$$

Now

$$
\begin{aligned}
\psi\left(k^{-2}-k\right) & =\frac{1}{k^{-2}-k}\left(k^{2}-\left(2 k-k^{-2}\right)^{2}\right) \\
& =\frac{1}{k^{2}\left(1-k^{3}\right)}\left(k^{6}-\left(2 k^{3}-1\right)^{2}\right) \\
& =\frac{1}{k^{2}}\left(3 k^{3}-1\right) \\
& =2 k^{4}+\frac{1}{k^{2}}\left(2 k^{3}-1\right)\left(1-k^{3}\right) . \\
& \geqq 2 k^{4}
\end{aligned}
$$

since $2^{-1 / 4}<k<1$. 
Similarly

$$
\begin{aligned}
\psi\left(k^{-2}-k^{2}\right) & =\frac{1}{k^{-2}-k^{2}}\left(k^{2}-\left(2 k^{2}-k^{-2}\right)^{2}\right) \\
& =\frac{1}{k^{2}\left(1-k^{4}\right)}\left(k^{6}-\left(2 k^{4}-1\right)^{2}\right) \\
& =2 k^{4}+\frac{1}{k^{2}\left(1-k^{4}\right)}\left(k^{6}-\left(2 k^{4}-1\right)^{2}-2 k^{6}\left(1-k^{4}\right)\right) \\
& =2 k^{4}+\frac{1}{k^{2}\left(1-k^{4}\right)}\left(2 k^{4}-1\right)\left(k^{6}-2 k^{4}+1\right) \\
& \geqq 2 k^{4}
\end{aligned}
$$

since $2 k^{4}-1>0$ and $k^{6}-2 k^{4}+1=\left(1-k^{2}\right)\left(1+k^{2}-k^{4}\right)>0$.

Thus

$$
\frac{\|g\|}{\|f\|} \geqq 2 k^{4}=\frac{2}{\|T\|^{2}}
$$

Lemma 2.4. Suppose $t(x)^{2} \neq t\left(x^{2}\right)$. Then

$$
T x=\alpha t(x)+\beta t(x) u+f
$$

where $u=t\left(x^{2}\right) t(x)^{-2} \in G_{2}$ has order $2, f \in l_{1}\left(G_{2}\right)$ does not contain terms in $t(x)$ or $t(x) u$, and

$$
\begin{aligned}
|\alpha|+|\beta| & >1.29 \\
\|f\| & <0.13 \\
|\alpha \bar{\beta}+\bar{\alpha} \beta| & <0.017 \\
\left|\frac{\bar{\alpha} \beta}{|\alpha|}+\frac{\alpha \bar{\beta}}{|\beta|}\right| & <0.60 .
\end{aligned}
$$

Proof. Writing $u=t\left(x^{2}\right) t(x)^{-2}$ we can expand $T x$ in the form $\alpha t(x)+\beta t(x) u+f$. We have immediately

$$
\begin{aligned}
& |\alpha|^{2}+|\beta|^{2}+\|f\|_{2}^{2}=1 \\
& |\alpha|+|\beta|+\|f\| \leqq\|T\|
\end{aligned}
$$

using Proposition 2.2. In addition the coefficient of $t\left(x^{2}\right)=t(x)^{2} u$ in $(T x)^{2}$ is at least $\|T\|^{-1}$ and so 


$$
2|\alpha \beta|+\|f\|_{2}^{2} \geqq\|T\|^{-1}
$$

since if $f=\sum_{y \in G_{2}} \gamma(y) y$, then the coefficient of $t(x)^{2} u$ is

$$
2 \alpha \beta+\sum_{y \in G_{2}} \gamma(y) \gamma\left(t(\dot{x})^{2} u y^{-1}\right)
$$

and $\left|\Sigma_{y \in G_{2}} \gamma(y) \gamma\left(t(x)^{2} u y^{-1}\right)\right| \leqq\|f\|_{2}^{2}$ by Cauchy's inequality.

First we note that from (1) and (3)

$$
(|\alpha|-|\beta|)^{2} \leqq 1-\|T\|^{-1}<0.30
$$

so that

$$
|\alpha|-|\beta|<0.55
$$

Now let $A=|\alpha|+|\beta|$. Then from (1) and (3)

$$
A^{2}+2\|f\|_{2}^{2} \geqq 1+\|T\|^{-1}>1+\frac{1}{\sqrt{2}}
$$

and as

$$
\|f\|_{2} \leqq\|T x\|-A<\sqrt{2}-A
$$

we have

i.e.

or

$$
\begin{aligned}
A^{2}+2(\sqrt{2}-A)^{2} & >1+\frac{1}{\sqrt{2}} \\
3 A^{2}-4 \sqrt{2} A+4 & >1+\frac{1}{\sqrt{2}} \\
\left(A-\frac{2 \sqrt{2}}{3}\right)^{2} & >\frac{1}{3 \sqrt{2}}-\frac{1}{9} .
\end{aligned}
$$

Therefore either

$$
\begin{aligned}
A & >\frac{2 \sqrt{2}}{3}+\frac{1}{3} \sqrt{\left(\frac{3}{\sqrt{2}}-1\right)} \\
& >\frac{2}{3}(1 \cdot 41)+\frac{1}{3}(1 \cdot 05)=1 \cdot 29
\end{aligned}
$$


or

$$
\begin{aligned}
A & <\frac{2 \sqrt{2}}{3}-\frac{1}{3} \sqrt{\left(\frac{3}{\sqrt{2}}-1\right)} \\
& <\frac{2}{3}(1 \cdot 42)-\frac{1}{3}(1 \cdot 04)=\cdot 60 .
\end{aligned}
$$

Since $A>|\alpha|>0 \cdot 70$, we have $A>1 \cdot 29$. Thus we have established (i).

For (ii) note that $\|f\|<\|T\|-1.29<1.42-1.29=0.13$ from (2).

Next, we note that since $T$ is a ${ }^{*}$-map

$$
T x^{-1}=\bar{\alpha} t(x)^{-1}+\bar{\beta} t(x)^{-1} u^{-1}+f^{*} .
$$

Multiplying by $T x$, we consider the coefficient of $u^{-1}$, which must be zero. If $u^{2} \neq e_{2}$, we obtain that

$$
\begin{aligned}
|\alpha \bar{\beta}| & \leqq|\beta|\left\|f^{*}\right\|+\|f\| \cdot\left\|f^{*}\right\| \\
0 \cdot 70|\beta| & \leqq 0 \cdot 13|\beta|+0 \cdot 02
\end{aligned}
$$

from which we conclude $|\beta| \leqq 0 \cdot 04$.

However $|\alpha|+|\beta|>1.29$ and $|\alpha|-|\beta|<0.55$ so that $|\beta|>0.37$ and so we have a contradiction. Hence $u^{2}=e_{2}$. Again identifying coefficients of $u$

$$
|\alpha \bar{\beta}+\beta \bar{\alpha}| \leqq\|f\| \cdot\left\|f^{*}\right\|<0 \cdot 017
$$

Finally

$$
\begin{aligned}
\left|\frac{\bar{\alpha} \beta}{|\alpha|}+\frac{\alpha \bar{\beta}}{|\beta|}\right| & \leqq\left|\frac{\bar{\alpha} \beta}{|\alpha|}-\frac{\bar{\alpha} \beta}{|\beta|}\right|+\left|\frac{\bar{\alpha} \beta}{|\beta|}+\frac{\alpha \bar{\beta}}{|\beta|}\right| \\
& \leqq|\alpha|-|\beta|+|\beta|^{-1}|\bar{\alpha} \beta+\alpha \bar{\beta}| \\
& \leqq 0.55+\frac{0.017}{0.37}<0.60 .
\end{aligned}
$$

Lemma 2.5. The set $\left\{t\left(x^{2}\right) t(x)^{-2}: x \in G_{1}\right\}$ contains at most one element $u \neq e_{2}$.

Proof. Suppose $t\left(x^{2}\right) t(x)^{-2}=u \neq e_{2}$ and $t\left(y^{2}\right) t(y)^{-2}=v \neq e_{2}$ with $v \neq u$. Then write

$$
\begin{aligned}
& T x=\alpha_{1} t(x)+\beta_{1} t(x) u+\gamma_{1} t(x) v+\delta_{1} t(x) u v+f \\
& T y=\alpha_{2} t(y)+\beta_{2} t(y) v+\gamma_{2} t(y) u+\delta_{2} t(y) u v+g
\end{aligned}
$$


where the terms are disjoint $\left(\gamma_{1}, \gamma_{2}, \delta_{1}\right.$ and $\delta_{2}$ may be zero). By our assumptions, $t(x) t(y), t(x) t(y) u, t(x) t(y) v, t(x) t(y) u v$ are distinct (note that $u$ and $v$ have order 2 by the preceding lemma). The sum of all terms in the product

$$
\left(\gamma_{1} t(x) v+\delta_{1} t(x) u v+f\right) *\left(\gamma_{2} t(y) u+\delta_{2} t(y) u v+g\right)
$$

may be estimated by $(0 \cdot 13)^{2}<0 \cdot 017$. Hence by considering each of the four elements above, we obtain

$$
\begin{aligned}
\|T x y\| & \geqq\left|\alpha_{1} \alpha_{2}+\beta_{1} \gamma_{2}+\gamma_{1} \beta_{2}\right|+\left|\alpha_{1} \gamma_{2}+\beta_{1} \alpha_{2}+\delta_{1} \beta_{2}\right|+\left|\alpha_{1} \beta_{2}+\beta_{1} \delta_{2}+\gamma_{1} \alpha_{2}\right| \\
& +\left|\alpha_{1} \delta_{2}+\beta_{1} \beta_{2}+\delta_{1} \alpha_{2}\right|-0 \cdot 017 \\
\geqq & \left|\alpha_{1} \alpha_{2}\right|\left(1+\operatorname{Re}\left(\frac{\beta_{1} \gamma_{2}}{\alpha_{1} \alpha_{2}}+\frac{\gamma_{1} \beta_{2}}{\alpha_{1} \alpha_{2}}\right)\right)+\left|\beta_{1} \alpha_{2}\right|\left(1+\operatorname{Re}\left(\frac{\alpha_{1} \gamma_{2}}{\beta_{1} \alpha_{2}}+\frac{\delta_{1} \beta_{2}}{\beta_{1} \alpha_{2}}\right)\right) \\
& +\left|\alpha_{1} \beta_{2}\right|\left(1+\operatorname{Re}\left(\frac{\beta_{1} \delta_{2}}{\alpha_{1} \beta_{2}}+\frac{\gamma_{1} \alpha_{2}}{\alpha_{1} \beta_{2}}\right)\right)+\left|\beta_{1} \beta_{2}\right|\left(1+\operatorname{Re}\left(\frac{\alpha_{1} \delta_{2}}{\beta_{1} \beta_{2}}+\frac{\delta_{1} \alpha_{2}}{\beta_{1} \beta_{2}}\right)\right) \\
\geqq & \left(\left|\alpha_{1}\right|+\left|\beta_{1}\right|\right)\left(\left|\alpha_{2}\right|+\left|\beta_{2}\right|\right)+\operatorname{Re} \gamma_{1}\left(\frac{\left|\alpha_{1} \alpha_{2}\right|}{\alpha_{1} \alpha_{2}} \beta_{2}+\frac{\left|\alpha_{1} \beta_{2}\right|}{\alpha_{1} \beta_{2}} \alpha_{2}\right) \\
& +\operatorname{Re} \gamma_{2}\left(\frac{\left|\beta_{1} \alpha_{2}\right|}{\beta_{1} \alpha_{2}} \alpha_{1}+\frac{\left|\alpha_{1} \alpha_{2}\right|}{\alpha_{1} \alpha_{2}} \beta_{1}\right)+\operatorname{Re} \delta_{1}\left(\frac{\left|\beta_{1} \alpha_{2}\right|}{\beta_{1} \alpha_{2}} \beta_{2}+\frac{\left|\beta_{1} \beta_{2}\right|}{\beta_{1} \beta_{2}} \alpha_{2}\right) \\
& +\operatorname{Re} \delta_{2}\left(\frac{\left|\alpha_{1} \beta_{2}\right|}{\alpha_{1} \beta_{2}} \beta_{1}+\frac{\left|\beta_{1} \beta_{2}\right|}{\beta_{1} \beta_{2}} \alpha_{1}\right)-0 \cdot 017 .
\end{aligned}
$$

Now

$$
\left|\frac{\left|\alpha_{1} \alpha_{2}\right|}{\alpha_{1} \alpha_{2}} \beta_{2}+\frac{\left|\alpha_{1} \beta_{2}\right|}{\alpha_{1} \beta_{2}} \alpha_{2}\right|=\left|\frac{\bar{\alpha}_{2} \beta_{2}}{\left|\alpha_{2}\right|}+\frac{\bar{\beta}_{2} \alpha_{2}}{\left|\beta_{2}\right|}\right|<0.60
$$

by Lemma 2.4 , and we can estimate each term similarly.

Hence

$$
\begin{aligned}
\|T x y\| & \geqq\left(\left|\alpha_{1}\right|+\left|\beta_{1}\right|\right)\left(\left|\alpha_{2}\right|+\left|\beta_{2}\right|\right)-0 \cdot 60\left(\left|\gamma_{1}\right|+\left|\gamma_{2}\right|+\left|\delta_{1}\right|+\left|\delta_{2}\right|\right) \\
& -0.017 \\
\geqq & (1 \cdot 29)^{2}-0 \cdot 60(0 \cdot 26)-0.017 \\
> & 1.66-0.16-0.02=1.48
\end{aligned}
$$

which is a contradiction, since $\|T x y\|<\sqrt{2}$.

We now come to the basic theorem. 
THEOREM 2.6. Let $G_{1}$ and $G_{2}$ be abelian groups with identities $e_{1}$ and $e_{2}$, and let $T: l_{1}\left(G_{1}\right) \rightarrow l_{1}\left(G_{2}\right)$ be an algebra homomorphism such that $T e_{1}=e_{2}$. Suppose $\|T\|<\sqrt{2}$; then if

(a) $\|T\|<\frac{1}{2}(1+\sqrt{3})$, $T$ takes the form

$$
T x=\psi(x) s(x)
$$

where $\psi \in \hat{G}_{1}$ and $s: G_{1} \rightarrow G_{2}$ is a homomorphism, and then $T$ is an isometry;

or (b) $\|T\| \geqq \frac{1}{2}(1+\sqrt{3})$, $T$ takes the form

$$
T x=\psi(x)\left(\frac{1}{2}(1+\varphi(x)) s(x)+\frac{1}{2}(1-\varphi(x)) s(x) u\right)
$$

where $\varphi, \psi \in \hat{G}_{1}$ and $\varphi$ has odd order, $s: G_{1} \rightarrow G_{2}$ is a homomorphism and $u \in G_{2}$ is an element of order 2 . In this case $\|T\|=\sqrt{2} \cos \pi / 4 n$, where $n$ is the order of $\varphi$. In both cases, if $T$ is a monomorphism, then $s$ is a monomorphism, and if $T$ is an isomorphism then so is $s$.

COROllaRy 2.7. If there is an algebra isomorphism $T$ : $l_{1}\left(G_{1}\right) \rightarrow l_{1}\left(G_{2}\right)$ with $\|T\|<\sqrt{ } 2$, then $G_{1}$ and $G_{2}$ are isomorphic.

REMARK. Example 1 shows that $\sqrt{2}$ is the best possible constant in the Theorem and its Corollary. Example 2 shows that $\frac{1}{2}(1+\sqrt{3})$ is best possible for (a). Example 4 shows that in case (b) $T$ can be an epimorphism while $s$ is not an epimorphism.

Proof of Theorem 2.6. Let $\epsilon_{2}$ denote the identity character on $G_{2}$. Then $\epsilon_{2} \circ T$ is a nonzero character $\psi$ on $G_{1}$ (since $T e_{1}=e_{2}$ ). If we define $S: l_{1}\left(G_{1}\right) \rightarrow l_{1}\left(G_{1}\right)$ by $S x=\psi^{-1}(x) x$ then $S$ is algebra automorphism, and so by considering $T S$ in place of $T$ we may reduce the problem to supposing $\epsilon_{2} \circ T=\epsilon_{1}$.

Using the notation of the preceding lemmas, suppose first that $t(x)^{2}=t\left(x^{2}\right)$ for all $x \in G_{1}$. Then by Lemma 2.3 if

then

$$
\begin{aligned}
T x & =\alpha t(x)+f \\
T x^{2^{n}} & =\beta t(x)^{2^{n}}+g
\end{aligned}
$$

where $\|g\| \geqq\left(2 /\|T\|^{2}\right)^{n}\|f\|$. Since $2>\|T\|^{2}$, we conclude that $f=0$ and hence $T x=\alpha t(x)$ where $\alpha=\alpha(x)$. Since $\epsilon_{2} \circ T=\epsilon_{1}, \alpha \equiv 1$, i.e. $T x=$ $t(x)$ and $t$ is a homomorphism. We thus obtain the result of (a).

Next suppose $\left\{t(x)^{-2} t\left(x^{2}\right): x \in G\right\}=\left\{e_{2}, u\right\}$ where $u$ is an element of order 2. Let $H=\left\{e_{2}, u\right\}$, and let $\pi: G_{2} \rightarrow G_{2} / H$ be the quotient 
map. Denote by $P: l_{1}\left(G_{2}\right) \rightarrow l_{1}\left(G_{2} / H\right)$ the induced algebra epimorphism, and consider $P T: l_{1}\left(G_{1}\right) \rightarrow l_{1}\left(G_{2} / H\right)$. As $\|P T\| \leqq\|T\|<\sqrt{ } 2$, we can appeal to the preceding lemmas. Let $\tilde{t}: G_{1} \rightarrow G_{2} / H$ be the map defined before Lemma 2.3. The mass of $T x$ concentrated in the coset $\tilde{f}(x)$ is at least $\|T\|^{-1}$, and hence as $2\|T\|^{-1}>\|T\|$, it follows that $\pi t(x)=\tilde{f}(x)$. Thus $\tilde{t}\left(x^{2}\right)=\pi t\left(x^{2}\right)=\tilde{t}(x)^{2}$ since $u \in H$, and so by the preceding paragraph

$$
P T x=\tilde{t}(x)
$$

and $\tilde{t}: G_{1} \rightarrow G_{2} / H$ is a homomorphism.

Thus for $x \in G_{1}$

$$
T x=\frac{1+\theta(x)}{2} t(x)+\frac{1-\theta(x)}{2} t(x) u+f
$$

where $f=\left(e_{2}-u\right) * \sum_{1}^{\infty} \gamma_{t} y_{t}$, and there is precisely one $y_{t}$ in each coset of $H$. Then $\|f\|=2 \Sigma\left|\gamma_{i}\right|<\|T\|-1$ and hence $\|f\|_{2}^{2}=2 \Sigma\left|\gamma_{i}\right|^{2}<$ $\frac{1}{2}(\|T\|-1)^{2}$. However

$$
\frac{1}{2}+\frac{1}{2}|\theta(x)|^{2}+\|f\|_{2}^{2}=1
$$

so that

$$
|\theta(x)|^{2} \geqq 1-(\|T\|-1)^{2}=2\|T\|-\|T\|^{2}
$$

Now suppose

$$
T x^{2}=\frac{1+\theta\left(x^{2}\right)}{2} t\left(x^{2}\right)+\frac{1-\theta\left(x^{2}\right)}{2} t\left(x^{2}\right) u+g .
$$

Then

$$
\|g\| \geqq 2\left\|f *\left(\frac{1+\theta(x)}{2} t(x)+\frac{1-\theta(x)}{2} t(x) u\right)\right\|-\|f\|^{2}
$$

and since $f=\frac{1}{2}\left(e_{2}-u\right) * f$

$$
\begin{aligned}
\|g\| & \geqq 2\|\theta(x) t(x) * f\|-\|f\|^{2} \\
& =2|\theta(x)|\|f\|-\|f\|^{2} \\
& \geqq\|f\|\left(2 \vee\left(2\|T\|-\|T\|^{2}\right)-\|T\|+1\right) \\
& =K\|f\|
\end{aligned}
$$

where $K=1+2 \sqrt{\left(2\|T\|-\|T\|^{2}\right)}-\|T\|>1$ since $\|T\|<8 / 5$. 
Thus it follows that $\left\|T x^{2^{n}}\right\| \geqq K^{n}\|f\|$, and hence that $f=0$, i.e. $T$ has the form

$$
T x=\frac{1}{2}(1+\theta(x)) t(x)+\frac{1}{2}(1-\theta(x)) t(x) u .
$$

By multiplying out, it is easy to see that

$$
\theta(x y)= \pm \theta(x) \theta(y)
$$

and hence that $\theta^{2}$ is a character on $G_{1}$. Since $\|T\|<\sqrt{2},|\theta(x) \pm i| \geqq$ $\epsilon>0$ for all $x \in G_{1}$ and some $\epsilon>0$. Hence $\theta^{2}$ has odd order, $n$, say. Define $\varphi=\left(\theta^{2}\right)^{(n+1) / 2}$; then $\varphi$ is also a character of order $n$, since $(n,(n+1) / 2)=1$. Now $\varphi(x)=\theta^{n+1}(x)= \pm \theta(x)$, and hence by defining $s(x)=t(x)$ or $t(x) u$ we may express $T$ in the form

$$
T x=\frac{1}{2}(1+\varphi(x)) s(x)+\frac{1}{2}(1-\varphi(x)) s(x) u .
$$

Since $\varphi$ is a character it follows easily that $s$ is a homomorphism. The norm of $T$ is then

$$
\begin{aligned}
\sup _{x \in G_{1}} & \frac{1}{2}(|1+\varphi(x)|+|1-\varphi(x)|) \\
& =\max _{1 \leqq k \leqq n}\left(\left|\cos \frac{k \pi}{n}\right|+\left|\sin \frac{k \pi}{n}\right|\right) \\
& =\max _{1 \leqq k \leqq n} \sqrt{2} \sin \left(\frac{k \pi}{n}+\frac{\pi}{4}\right) \\
& =\sqrt{2} \cos \frac{\pi}{4 n} .
\end{aligned}
$$

If $n=3$

$$
\sqrt{2} \cos \frac{\pi}{4 n}=\frac{1+\sqrt{3}}{2} \text {. }
$$

If $T$ is a monomorphism in case (b) (case (a) is trivial), let $M \subset G_{2}$ be the set such that $s(M)=e_{2}$. Then $T m \in \operatorname{lin}\left(e_{2}, u\right)$ for $m \in M$, and so $M$ has at most two members. If $M \neq\left\{e_{1}\right\}$, there exists $v \in M$ and $v^{2}=$ $e_{1}$. Hence $\varphi(v)=+1$ since $\varphi$ is of odd order and so $T v=\psi(v) e_{2}$, contradicting the fact that $T$ is a monomorphism.

If $T$ is an isomorphism, let $N=s\left(G_{1}\right)$. If $u \notin N$ then there must exist $x \in G_{1}$ such that $s(x) u=u$ and $1-\varphi(x) \neq 0$. But then $s(x)=e_{1}$ and by the result for monomorphisms, $x=e_{1}, \varphi(x)=1$. Thus $u \in N$ and so $T x \in l_{1}\left(s\left(G_{1}\right)\right)$ for $x \in G_{1}$. Thus $s\left(G_{1}\right)=G_{2}$ since $T$ is onto. 
3. General case. We now use the results for discrete groups to establish the corresponding theorems for locally compact abelian groups.

First consider homomorphisms from $L^{1}\left(G_{1}\right)$ into $M\left(G_{2}\right)$. If $I$ is such a homomorphism, then $T$ possesses a unique extension $\bar{T}: M\left(G_{1}\right) \rightarrow M\left(G_{2}\right)$ which is continuous on bounded sets from the strong operator topology (of $M\left(G_{1}\right)$ operating on $L^{1}\left(G_{1}\right)$ ) to the weak*-topology (of $M\left(G_{2}\right)=C_{0}\left(G_{2}\right)^{*}$ ) (see [4] Theorem 4.1.1 and Remark afterwards). We also observe that in the strong-operator topology, the unit ball of $M\left(G_{1}\right)$ is the closed absolutely convex cover of the Dirac measures $\left\{\delta_{x}: x \in G_{1}\right\}$ (see [4] Lemma 1.1.3). Hence $\bar{T}$ is uniquely determined by its behaviour on the atomic measures, which we identify with $l_{1}\left(G_{1}\right)$.

THEOREM 3.1. Let $G_{1}$ and $G_{2}$ be locally compact abelian groups and $T: L^{1}\left(G_{1}\right) \rightarrow M\left(G_{2}\right)$ be an algebra homomorphism such that $\|T\|<\sqrt{2}$ and $\bar{T} \delta_{e_{1}}=\delta_{e_{2}}$. Then

(i) if $\|T\|<\frac{1}{2}(1+\sqrt{3})$, then $\|T\|=1$ and $T$ takes the form

$$
\widehat{T f}(\chi)=\hat{f}(\hat{s}(\chi) \psi) \quad\left(\chi \in \hat{G}_{2}\right)
$$

where $\psi \in \hat{G}_{1}$ and $s: G_{1} \rightarrow G_{2}$ is a continuous homomorphism;

(ii) if $\|T\| \geqq \frac{1}{2}(1+\sqrt{3})$, then $T$ takes the form

$$
\begin{aligned}
& \widehat{T f}(\chi)=\hat{f}(\hat{s}(\chi) \psi) \quad \text { if } \quad \chi(u)=1 \\
& =\hat{f}(\hat{s}(\chi) \psi \varphi) \text { if } \quad \chi(u)=-1
\end{aligned}
$$

where $\varphi, \psi \in \hat{G}_{1}, \varphi$ is of odd order $n, s: G_{1} \rightarrow G_{2}$ is a continuous homomorphism, and $u \in G_{2}$ is an element of order 2 . Then $\|T\|=$ $\sqrt{2} \cos \pi / 4 n$.

Proof. Let $P: M\left(G_{2}\right) \rightarrow l_{1}\left(G_{2}\right)$ be the natural projection; $P$ is an algebra homomorphism. Consider $P \bar{T}: l_{1}\left(G_{1}\right) \rightarrow l_{1}\left(G_{2}\right)$. Clearly $\|\bar{T}\|=$ $\|T\|$ and $\|P\|=1$, and so $\|P \bar{T}\|<\sqrt{2}$. By Theorem 2.6, either

(i) $P \bar{T} \delta_{x}=\psi(x) \delta_{s(x)}$

or (ii) $P \bar{T} \delta_{x}=\psi(x)\left[\frac{1}{2}(1+\varphi(x)) \delta_{s(x)}+\frac{1}{2}(1-\varphi(x)) \delta_{s(x) u}\right]$.

Consider case (i). Then

$$
\bar{T} \delta_{x}=\psi(x) \delta_{s(x)}+\mu
$$

where $\mu$ is nonatomic, and $\|\mu\|<\sqrt{2}-1$.

$$
\bar{T} \delta_{x^{2}}=\psi\left(x^{2}\right) \delta_{s\left(x^{2}\right)}+\nu
$$

where $\nu$ is nonatomic and 


$$
\begin{aligned}
\|\nu\| & \geqq\left\|2 \psi(x) \delta_{s(x)} * \mu\right\|-\|\mu * \mu\| \\
& \geqq(2-\|\mu\|)\|\mu\| \\
& \geqq(3-\sqrt{2})\|\mu\| .
\end{aligned}
$$

By iteration we obtain a contradiction (cf. 2.6) unless $\mu=0$, since $3-\sqrt{2}>1$.

Thus

$$
\bar{T} \delta_{x}=\psi(x) \delta_{s(x)}
$$

On the Dirac measures, both the strong-operator and weak*topologies agree with the group topologies, so that $s$ and $\psi$ are continuous. The form of $T$ now follows from the remarks preceding the Theorem, and replacing $\psi$ by $\psi^{-1}$.

For case (ii) we compose with the quotient $Q: M\left(G_{2}\right) \rightarrow M\left(G_{2} / H\right)$ where $H=\left\{e_{2}, u\right\}$ is a subgroup of $G_{2}$. Then

$$
P^{\prime} Q \bar{T} \delta_{x}=\psi(x) \delta_{\pi s(x)}
$$

where $P^{\prime}$ is the natural map $P^{\prime}: M\left(G_{2} / H\right) \rightarrow l_{1}\left(G_{2} / H\right)$, and $\pi$ is the quotient map $\pi: G_{2} \rightarrow G_{2} / H$. As above $\psi$ and $\pi s$ are continuous and

Thus

$$
Q \bar{T} \delta_{x}=\psi(x) \delta_{\pi s(x)}
$$

$$
\bar{T} \delta_{x}=P \bar{T} \delta_{x}+\mu
$$

where $\mu$ is nonatomic and satisfies $\mu=\frac{1}{2}\left(\delta_{e_{2}}-\delta_{u}\right) * \mu$. Then

$$
\bar{T} \delta_{x^{2}}=P \bar{T} \delta_{x^{2}}+\nu
$$

where $\nu$ is nonatomic and satisfies

$$
\begin{aligned}
\nu & =\psi(x)\left[(1+\varphi(x)) \mu * \delta_{s(x)}+(1-\varphi(x)) \mu * \delta_{s(x) u}\right]+\mu * \mu \\
& =2 \psi(x) \varphi(x) \mu * \delta_{s(x)}+\mu * \mu .
\end{aligned}
$$

Thus

$$
\begin{aligned}
\|\nu\| & \geqq 2\|\mu\|-\|\mu\|^{2} \\
& \geqq(3-\sqrt{ } 2)\|\mu\|
\end{aligned}
$$

and arguing as before $\mu=0$, i.e.

$$
\bar{T} \delta_{x}=P \bar{T} \delta_{x}
$$


Now

$$
\bar{T} \delta_{x}=\psi(x)\left[\frac{1}{2}(1+\varphi(x)) \delta_{s(x)}+\frac{1}{2}(1-\varphi(x)) \delta_{s(x) u}\right]
$$

where $\psi$ is continuous. To prove $\varphi$ continuous, since $\varphi$ has finite order, it is sufficient to establish that $\{x: \varphi(x)=1\}$ is closed. Suppose $x_{\alpha} \rightarrow x$ and $\varphi\left(x_{\alpha}\right)=1$. Then

$$
\bar{T} \delta_{x_{\alpha}}=\psi\left(x_{\alpha}\right) \delta_{s\left(x_{\alpha}\right)}
$$

so that

$$
\bar{T} \delta_{x}=\psi(x) \delta_{z}
$$

where $s\left(x_{\alpha}\right) \rightarrow z$. Hence $\varphi(x)= \pm 1$; but $\varphi$ has odd order so that $\varphi(x)=1$ and $\varphi$ is continuous. It follows that $s$ is also continuous. The general form of $T$ follows as above, again replacing $\psi$ by $\psi^{-1}$.

The proof above clearly shows that a homomorphism $T$ of $M\left(G_{1}\right)$ into $M\left(G_{2}\right)$ with $\|T\|<\sqrt{2}$ and $T \delta_{e_{1}}=\delta_{e_{2}}$ has the form on atomic measures given by either

(1) $T \delta_{x}=\psi(x) \delta_{s(x)}$, or

(2) $T \delta_{x}=\psi(x)\left[\frac{1}{2}(1+\varphi(x)) \delta_{s(x)}+\frac{1}{2}(1-\varphi(x)) \delta_{s(x) u}\right]$

with $\psi, \varphi, s$ and $u$ as before.

However we cannot deduce that $s$ is continuous or the form of $T$ on the nonatomic measures. For example, (see [8], 3.4.1) let $G$ be a compact infinite group and let $G_{d}$ be the same group with the discrete topology. Then the algebra homomorphism $P: M(G) \rightarrow l_{1}(G)=$ $M\left(G_{d}\right)$ as in the proof of the theorem has the above form, but the identity map is not continuous.

Corollary 3.2. Suppose $T$ is an algebra isomorphism of $L^{1}\left(G_{1}\right)$ onto $L^{1}\left(G_{2}\right)$ [resp: $M\left(G_{1}\right)$ onto $M\left(G_{2}\right)$ ] with $\|T\|<\sqrt{2}$. Then $G_{1}$ and $G_{2}$ are isomorphic.

Proof. By [8] 4.6.4 it is sufficient to prove the result in the $L^{1}$-case. In this case $\bar{T}$ is also an isomorphism and satisfies $\bar{T} \delta_{e_{1}}=$ $\delta_{e_{2}}$. By Theorem 3.1 and Theorem 2.6 there is an isomorphism $s: G_{1} \rightarrow G_{2}$ and $s$ is continuous.

Since $T^{-1}$ is onto $L^{1}\left(G_{1}\right), T^{-1}$ has a unique extension to $M\left(G_{2}\right)$ (without any continuity requirements). Thus $\bar{T}^{-1}$ is the unique extension and is hence continuous for the strong operator and weak*. topologies on bounded sets.

The form of $\bar{T}$ is either

(1) $\bar{T} \delta_{x}=\psi(x) \delta_{s(x)}$

or

(2) $\bar{T} \delta_{x}=\psi(x)\left[\frac{1}{2}(1+\varphi(x)) \delta_{s(x)}+\frac{1}{2}(1-\varphi(x)) \delta_{s(x) u}\right]$. 
Thus either

or

(1) $\bar{T}^{-1} \delta_{x}=\psi^{-1}\left(s^{-1}(x)\right) \delta_{s^{-1}(x)}$

(2) $\bar{T}^{-1} \delta_{x}=\psi^{-1}\left(s^{-1}(x)\right)$

$$
\times\left[\frac { 1 } { 2 } \left(1+\varphi^{-1}\left(s^{-1}(x)\right) \delta_{s^{-1}(x)}+\frac{1}{2}\left(1-\varphi^{-1}\left(s^{-1}(x)\right) \delta_{s^{-1}(x u)}\right] .\right.\right.
$$

[Note that $\varphi\left(s^{-1}(u)\right)=1$ since $u^{2}=e_{2}$ and $\varphi$ is of odd order.]

The continuity of $s^{-1}$ follows as in Theorem 3.1.

Theorem 3.1 is false if $\bar{T} \delta_{e_{1}} \neq \delta_{e_{2}}$ (see Example 3). However with a stronger condition on the norm, we have the following result, essentially due to Saeki [9] (see [8] 4.6.3.).

THEOREM 3.3. Let $G_{1}$ and $G_{2}$ be locally compact abelian groups and $T$ an algebra homomorphism of $L^{1}\left(G_{1}\right)$ into $M\left(G_{2}\right)$ with $\|T\|<$ $\frac{1}{2}(1+\sqrt{2})$. Then $\|T\|=1$ and $T$ has the factorization

$$
L^{1}\left(G_{1}\right) \stackrel{s}{\rightarrow} M\left(G_{2} / H_{2}\right) \stackrel{\pi^{*}}{\rightarrow} M\left(G_{2}\right)
$$

where $\mathrm{H}_{2}$ is a compact subgroup of $G_{2}$, s is a continuous homomorphism of $G_{1}$ into $G_{2} / H_{2}, \psi \in \hat{G}_{1}, \rho \in H_{2}$,

$$
\widehat{S f^{\prime}}(\chi)=\hat{f}(\hat{s}(\chi) \psi) \quad\left(\chi \in \widehat{G_{2} / H_{2}}\right)
$$

and $\pi: C_{0}\left(G_{2}\right) \rightarrow C_{0}\left(G_{2} / H_{2}\right)$ is defined by

$$
(\pi f)(\xi)=\int_{H_{2}} f(x y) \rho\left(y^{-1}\right) d m_{H_{2}}\left(y^{-1}\right) \quad\left(\xi=H_{2} x\right) .
$$

Proof. Let $\bar{T}$ be the extension of $T$ to $M\left(G_{1}\right)$ as in Theorem 3.1. Then $\bar{T} \delta_{e_{1}}$ is an idempotent in $M\left(G_{2}\right)$ with $\left\|\bar{T} \delta_{e_{1}}\right\|<\frac{1}{2}(1+\sqrt{2})$. By Saeki [9], $\left\|\tilde{T} \delta e_{1}\right\|=1$ and $\bar{T} \delta_{e_{1}}=\rho m_{H_{2}}$ where $H_{2}$ is a compact subgroup of $G_{2}$ and $\rho \in \hat{H}_{2}$, (see [4] Theorem 2.1.4). Since $\rho m_{H_{2}}$ is the identity for the image of $\bar{T}, T$ must factor through $M\left(G_{2} / H_{2}\right)$.

Let $L^{1}(G) \stackrel{s}{\rightarrow} M\left(G_{2} / H_{2}\right) \stackrel{\pi^{*}}{\rightarrow} M\left(G_{2}\right)$ be the factorization with $\pi$ defined as in the statement of the theorem. Then $S \delta_{e_{1}}=\delta_{\xi_{1}}$ where $\xi_{1}$ is the identity of $G_{2} / H_{2}$, and since $\left\|\pi^{*}\right\|=1,\|S\|<\frac{1}{2}(1+\sqrt{2})$. The result now follows from Theorem 3.1.

Finally in this section, we give the corresponding results for $C(G)$ and $L^{\infty}(G)$ with $G$ a compact group.

THEOREM 3.4. Let $G_{1}$ and $G_{2}$ be compact abelian groups and let $T$ be an algebra homomorphism of $C\left(G_{1}\right)$ into $C\left(G_{2}\right)$ with Tone-to-one and $\|T\|<\sqrt{2}$. Then there exists a group homomorphism sof $G_{2}$ into $G_{1}$ and $\psi \in \hat{G}_{2}$ such that either

(1) $\|T\|=1$ and $T$ has the form 


$$
(T f)(x)=\psi(x) f(s(x))
$$

or (2) $\|T\|=\sqrt{2} \cos \pi / 4 n$ for an odd number $n>1$ and $T$ has the form

$$
(T f)(x)=\psi(x)\left[\frac{1}{2}(1+\varphi(x)) f(s(x))+\frac{1}{2}(1-\varphi(x)) f(s(x) u)\right]
$$

for some $\varphi \in \hat{G}_{2}$ of order $n$ and $u \in G_{1}$, of order 2 .

Proof. The characters on a compact abelian group are the only idempotents of norm less than 2. Thus $T$ maps characters into characters. It follows that $(T f)\left(e_{2}\right)=f\left(e_{1}\right)$ for all $f \in C\left(G_{1}\right)$, and as in [16] Theorem 2, $T^{*}$ is a homomorphism of $M\left(G_{2}\right)$ into $M\left(G_{1}\right)$. Since $T^{*} \delta_{e_{2}}=\delta_{e_{1}}$ and $\left\|T^{*}\right\|<\sqrt{2}$, the result follows from Theorem 3.1.

Note that $s$ need not be an epimorphism (Example 4).

COROLlaRY 3.5. Suppose Tis an isomorphism of $C\left(G_{1}\right)$ onto $C\left(G_{2}\right)$ with $\|T\|<\sqrt{2}$. Then $G_{1}$ and $G_{2}$ are isomorphic.

Proof. This follows from Corollary 3.2.

Note that $\sqrt{2}$ is again the best possible constant (Example 1).

THEOREM 3.6. Let $G_{1}$ and $G_{2}$ be compact abelian groups and $T: L^{\infty}\left(G_{1}\right) \rightarrow L^{\infty}\left(G_{2}\right)$ be a one-one. algebra homomorphism. Then

(i) if $\|T\|<\frac{1}{2}(1+\sqrt{3})$

$$
T f(x)=\psi(x) f(s(x))
$$

where $s: G_{2} \rightarrow G_{1}$ is a continuous homomorphism and $\psi \in \hat{G}_{2}$

(ii) if $T$ is an isomorphism onto $L^{\infty}\left(G_{2}\right)$ and $\|T\|<\sqrt{2}$ then $G_{1}$ and $G_{2}$ are isomorphic.

Proof. As $T$ maps characters to characters, $T$ maps $C\left(G_{1}\right)$ to $C\left(G_{2}\right)$. If $T$ is an isomorphism then $T: C\left(G_{1}\right) \rightarrow C\left(G_{2}\right)$ is an isomorphism and case (ii) follows from 3.5. For case (i) we observe by 3.4 that

$$
T f(x)=\psi(x) f(s(x)) \quad f \in C\left(G_{1}\right)
$$

where $\psi \in \hat{G}_{2}$ and $s: G_{2} \rightarrow G_{1}$ is a continuous homomorphism. Let $H=\operatorname{ker} s$ and consider $P: L^{\infty}\left(G_{2}\right) \rightarrow L^{\infty}\left(G_{2} / H\right)$ the natural projection

$$
P \varphi[\pi(x)]=\int_{H} \varphi(x h) d m_{H}(h)
$$

where $m_{H}$ is the Haar measure on $H$, and $\pi$ the quotient map. 
Then PT: $L^{\infty}\left(G_{1}\right) \rightarrow L^{\infty}\left(G_{2} / H\right)$ is an algebra homomorphism and $P T\left(C\left(G_{1}\right)\right)=C\left(G_{2} / H\right)$. Hence for $g \in C\left(G_{2} / H\right), f \in L^{\infty}\left(G_{1}\right)$,

$$
P T f * g=P T\left(f *(P T)^{-1} g\right)=P \tilde{T} f * g
$$

where

$$
\tilde{T} f(x)=\psi(x) f(s(x))
$$

It follows that

$$
P T f=P \tilde{T} f
$$

and hence that $T f=\tilde{T} f+k$ where

$$
\int_{H} k(x y) d m_{H}(y)=0 \quad\left(x \in G_{2}\right) .
$$

Now $T(f * f)=\tilde{T}(f * f)$ and so

$$
2 \tilde{T} f * k+k * k=0
$$

$\tilde{T} f$ is constant on cosets of $H$ and so $\tilde{T} f * k=0$. Thus $k * k=0$ and $k=0$ since $G_{2}$ is abelian.

4. Non-abelian groups. In the non-abelian case, we cannot expect results about the form of near isometries. If $G$ is compact, but not abelian, there exist many isomorphisms of $C(G)$ [or $L^{1}(G)$ ] onto itself with norms arbitrarily close to one. An isometry can be perturbed in different ways by automorphisms of the minimal ideals. However we can still ask whether isomorphisms of the algebras determine isomorphisms of the groups. Again we begin with the discrete case. Let $G_{1}$ and $G_{2}$ be arbitrary groups and $T: l_{1}\left(G_{1}\right) \rightarrow l_{1}\left(G_{2}\right)$ be an algebra homomorphism with $\|T\|<\sqrt{2}$ and $T e_{1}=e_{2}$. By Proposition 2.1, the map $t: G_{1} \rightarrow G_{2}$ is well-defined. But Proposition 2 is false in the non-abelian case since $T$ need not be a ${ }^{*}$-map. If we impose a stronger condition on the norm of $T$, we get that $t$ is a homomorphism directly.

LEMMA 4.1. If $\|T\|<\lambda_{0}$ where $\lambda_{0}$ is the largest root of the equation $\lambda^{3}+\lambda^{2}-2 \lambda-1=0$, then $t$ is a homomorphism.

Proof. Let $T x=\alpha t(x)+f$ and $T y=\beta t(y)+g$ where $|\alpha| \geqq 1 /\|T\|$, $|\beta| \geqq 1 /\|T\|$, with $f$ and $g$ disjoint from $t(x)$ and $t(y)$ respectively. Then the modulus of the coefficient of $t(x) t(y)$ in Txy is greater than $|\alpha||\beta|-\|f\|\|g\|$. Now if $t(x) t(y) \neq t(x y)$, this must be less than $\|T\|-1 /\|T\|$. Thus we must have 


$$
\begin{aligned}
\|T\|-\frac{1}{\|T\|} & \geqq|\alpha\|\beta \mid-\| f\|\| g \| \\
& \geqq|\alpha \| \beta|-(\|T\|-|\alpha|)(\|T\|-|\beta|) \\
& =(|\alpha|+|\beta|)\|T\|-\|T\|^{2} \\
& \geqq 2-\|T\|^{2} .
\end{aligned}
$$

But this is impossible since $\|T\|<\lambda_{0}$ and $\lambda_{0}$ is the largest root of $\lambda-1 / \lambda=2-\lambda^{2}$.

Thus $t(x y)=t(x) t(y)$ for all $x, y \in G_{1}$ and so $t$ is a homomorphism.

Note. $\quad \lambda_{0} \sim 1 \cdot 247$.

With the condition $\|T\|<\sqrt{2}$, we can show that $t$ is not too far from a homomorphism in the following sense.

Lemma 4.2. For $x \in G_{1}$, the set $\{t(y) t(z): y z=x\}$ is finite;

Proof. If $T y=\alpha t(y)+f$ and $T z=\beta t(z)+g$ with $|\alpha| \geqq 1 /\|T\|$, $|\beta| \geqq 1 /\|T\|$ and $f$ and $g$ disjoint from $t(y)$ and $t(z)$ respectively, then the modulus of the coefficient of $t(y) t(z)$ in $T y z=T x$ is greater than

$$
\left|\alpha\|\beta \mid-\| f\|\| g\|\geqq 2-\| T \|^{2}\right. \text { as before. }
$$

Since this is positive and $\|T x\|<\sqrt{2}$, it follows that the set $\{t(y) t(z): y z=x\}$ is finite.

These two lemmas give corresponding results for locally compact groups. We give only the results for isomorphisms, though clearly there are slightly more general results.

THEOEREM 4.3. Let $G_{1}$ and $G_{2}$ be locally compact groups and $T$ an algebra isomorphism of $L^{1}\left(G_{1}\right)$ onto $L^{1}\left(G_{2}\right)$ with $\|T\|<\lambda_{0}$ where $\lambda_{0}$ is the largest root of the equation $\lambda^{3}+\lambda^{2}-2 \lambda-1=0$, then $G_{1}$ and $G_{2}$ are isomorphic.

Proof. By [4] $\S 4$, there is a unique extension $\tilde{T}$ of $T$ from $M\left(G_{1}\right)$ onto $M\left(G_{2}\right)$ which will also be an isomorphism with $\|\tilde{T}\|<\lambda_{0}$, and which is continuous on bounded sets as a map from the strong operator topology into the weak* topology.

Now restricting to the atomic measures on $G_{1}$ and using Lemma 4.1, we have an isomorphism $t: G_{1} \rightarrow G_{2}$. $t$ is continuous by the continuity of $\tilde{T}$ as in Theorem 3.1, so it remains only to prove that $t^{-1}$ is continuous. Suppose not. Then there exists a compact neighborhood $V$ of $e_{1}$ in $G_{1}$ such that $t(V)$ is not a neighborhood of $e_{2}$. By taking $U$ 
such that $U U^{-1} \subset V$ if necessary, we can assume that the measure of $t(V)$ is zero. Let $\chi_{V}$ denote the characteristic function of $V$ and $m_{1}$ the Haar measure on $G_{1}$. Then $\chi_{V} \in L^{1}\left(G_{1}\right)$ and by [4] Lemma 1.1.2, $\left(1 / m_{1}(V)\right) \chi_{V}$ can be approximated in the strong operator topology by elements in the convex hull of $\left\{\delta_{x}: x \in V\right\}$. Suppose $\sum_{i=1}^{n} \lambda_{l} \delta_{x_{i}}$ is such an element. Then for each $i, \tilde{T} \delta_{x_{i}}=\alpha\left(x_{i}\right) \delta_{t\left(x_{i}\right)}+\nu_{i}$ where $\left|\alpha\left(x_{i}\right)\right|>1 /\|T\|$, $\nu_{i}\left(\left\{t\left(x_{i}\right)\right\}\right)=0$ and $\left\|\nu_{l}\right\| \leqq\|T\|-1 /\|T\|$.

Then

$$
\tilde{T}\left(\sum_{1}^{n} \lambda_{l} \delta_{x_{i}}\right)=\sum_{1}^{n} \lambda_{i} \alpha\left(x_{t}\right) \delta_{t\left(x_{i}\right)}+\sum_{1}^{n} \lambda_{l} \nu_{i}
$$

Now $\left(1 / m_{1}(V)\right) T \chi_{V}$ is the $w^{*}$ limit of such elements. Thus $\left(1 / m_{1}(V)\right) T \chi_{V}=\mu+\nu$ where $\operatorname{supp}(\mu) \subset t(V)$ and $\|\nu\| \leqq\|T\|-1 /\|T\|$.

Since $t(V)$ has measure zero; $\mu$ is a singular measure. But $T \chi_{V} \in L^{1}\left(G_{2}\right)$ and so $\left\|\left(1 / m_{1}(V)\right) T \chi_{V}\right\| \leqq\|\nu\| \leqq\|T\|-1 /\|T\|$. But there exists a net of such $V$ such that $\left(1 / m_{1}(V)\right) \chi_{V}$ tends to $\delta_{e_{1}}$ in the strong operator topology. Therefore $\left(1 / m_{1}(V)\right) T \chi_{V} \rightarrow \delta_{e_{2}}$ in the $w^{*}$-topology, which is a contradiction, since $\left\|\left(1 / m_{1}(V)\right) T \chi_{V}\right\| \leqq\|T\|-1 /\|T\|<1$. Hence $t^{-1}$ is continuous and the result is proved.

Corollary 4.4. Let $G_{1}$ and $G_{2}$ be compact groups and $T$ an algebra isomorphism of $C\left(G_{1}\right)\left[L^{\infty}\left(G_{1}\right)\right]$ onto $C\left(G_{2}\right)\left[L^{\infty}\left(G_{2}\right)\right]$ with $\|T\|<$ $\lambda_{0}$. Then $G_{1}$ and $G_{2}$ are isomorphic.

Proof. By [12] p. 861, it is sufficient to prove the result for $T$ mapping $C\left(G_{1}\right)$ onto $C\left(G_{2}\right)$. The adjoint map $T^{*}$ is an algebra isomorphism of $M\left(G_{2}\right)$ onto $M\left(G_{1}\right)$ which maps $L^{1}\left(G_{2}\right)$ onto $L^{1}\left(G_{1}\right)$ ([4] Theorem 1). The result now follows from 4.3.

THEOREM 4.5. Let $G_{1}$ and $G_{2}$ be locally compact connected groups and $T$ an algebra isomorphism of $L^{1}\left(G_{1}\right)$ onto $L^{1}\left(G_{2}\right)$ with $\|T\|<$ $\sqrt{2}$. Then $G_{1}$ and $G_{2}$ are isomorphic.

Proof. As in the proof of $4.3, t$ is a continuous one-to-one map from $G_{1}$ onto $G_{2}$. By Lemma 4.2, for $x \in G_{1},\{t(y) t(z): y z=x\}$ is a finite set. But it is the image of $G_{1}$ under the continuous map $y \mapsto t(y) t\left(y^{-1} x\right)$. Since $G_{1}$ is connected, it is a one point set, and since $t\left(e_{1}\right)=e_{2}$, this point is $t(x)$. Thus $t$ is an isomorphism. The continuity of $t^{-1}$ follows as in 4.3 since $\|T\|-1 /\|T\|$ is still less than one.

CoROllary 4.6. Let $G_{1}$ and $G_{2}$ be connected compact groups and $T$ an algebra isomorphism of $C\left(G_{1}\right)\left[L^{\infty}\left(G_{1}\right)\right]$ onto $C\left(G_{2}\right)\left[L^{\infty}\left(G_{2}\right)\right]$ with $\|T\|<\sqrt{2}$. Then $G_{1}$ and $G_{2}$ are isomorphic. 


\section{REFERENCES}

1. D. Amir, On isomorphisms of continuous function spaces, Israel J. Math., 3 (1965), 205-210.

2. M. Cambern, A generalised Banach-Stone theorem, Proc. Amer. Math. Soc., 17 (1966), 396-400.

3. R. E. Edwards, Bipositive and isometric isomorphisms of some convolution algebras, Canad. J. Math., 17 (1965), 839-846.

4. F. P. Greenleaf, Norm decreasing homomorphisms of group algebras, Pacific J. Math., 15 (1965), $1187-1219$.

5. B. E. Johnson, Isometric ısomorphisms of measure algebras, Proc. Amer. Math. Soc., 15 (1964), 186-188.

6. S. K. Parrott, Isometric multipleers, Pacific J. Math., 25 (1968), 159-166.

7. R. Rigelhof, Norm-decreasing homomorphisms of group algebras, Trans. Amer. Math. Soc., 136 (1969), 361-372.

8. W. Rudin, Fourier Analysts on Groups, Interscience (New York) 1960.

9. S. Saeki, On norms of idempotent measures, Proc. Amer. Math. Soc., 19 (1968), 600-602.

10. — On norms of idempotent measures II, Proc. Amer. Math. Soc., 19 (1968), 367-371.

11. R. S. Strichartz, Isometric tsomorphisms of measure algebras, Pacific J. Math., 15 (1965), 315-317.

12. - Isomorphisms of group algebras, Proc. Amer. Math. Soc., 17 (1966), 858-862.

13. J. G. Wendel, Left centralizers and isomorphisms of group algebras, Pacific J. Math., 2 (1952), 251-261.

14. G. V. Wood, A note on isomorphisms of group algebras, Proc. Amer. Math. Soc., 25 (1970), 771-775.

15. - Isomorphisms of $L^{p}$ group algebras, J. London Math. Soc., 4 (1972), 425-428.

16. - Homomorphisms of group algebras, Duke Math. J., 41 (1974), 255-261.

Received June 30, 1975.

Department of Pure Mathematics

University College of Swansea

SINGLETON PARK

SWANSEA, SA2 8PP 


\section{PACIFIC JOURNAL OF MATHEMATICS}

\section{EDITORS}

RICHARD ARENS (Managing Editor)

University of California

Los Angeles, California 90024

\section{J. Dugundu}

Department of Mathematics University of Southern California Los Angeles, California 90007

D. Gilbarg and J. Milgram Stanford University Stanford, California 94305

\section{ASSOCIATE EDITORS}
E. F. BECKENBACH
B. H. NeumanN
F. WOLF
K. YoSHIDA

\section{SUPPORTING INSTITUTIONS}

UNIVERSITY OF BRITISH COLUMBIA CALIFORNIA INSTITUTE OF TECHNOLOGY

UNIVERSITY OF CALIFORNIA

MONTANA STATE UNIVERSITY

UNIVERSITY OF NEVADA

NEW MEXICO STATE UNIVERSITY

OREGON STATE UNIVERSITY

UNIVERSITY OF OREGON

OSAKA UNIVERSITY

\author{
UNIVERSITY OF SOUTHERN CALIFORNIA \\ STANFORD UNIVERSITY \\ UNIVERSITY OF HAWAII \\ UNIVERSITY OF TOKYO \\ UNIVERSITY OF UTAH \\ WASHINGTON STATE UNIVERSITY \\ UNIVERSITY OF WASHINGTON \\ AMERICAN MATHEMATICAL SOCIETY
}

The Supporting Institutions listed above contribute to the cost of publication of this Journal, but they are not owners or publishers and have no responsibility for its contents or policies.

Mathematical papers intended for publication in the Pacific Journal of Mathematics should be in typed form or offset-reproduced (not dittoed), double spaced with large margins. Underline Greek letters in red, German in green, and script in blue. The first paragraph or two must be capable of being used separately as a synopsis of the entire paper. Items of the bibliography should not be cited there unless absolutely necessary, in which case they must be identified by author and Journal, rather than by item number. Manuscripts, in duplicate, may be sent to any one of the four editors. Please classify according to the scheme of Math. Reviews, Index to Vol. 39. All other communications should be addressed to the managing editor, or Elaine Barth, University of California, Los Angeles, California, 90024.

100 reprints are provided free for each article, only if page charges have been substantially paid. Additional copies may be obtained at cost in multiples of 50 .

The Pacific Journal of Mathematics is issued monthly as of January 1966. Regular subscription rate: $\$ 72.00$ a year (6 Vols., 12 issues). Special rate: $\$ 36.00$ a year to individual members of supporting institutions.

Subscriptions, orders for back numbers, and changes of address should be sent to Pacific Journal of Mathematics, 103 Highland Boulevard, Berkeley, California, 94708.

PUBLISHED BY PACIFIC JOURNAL OF MATHEMATICS, A NON-PROFIT CORPORATION

Printed at Jerusalem Academic Press, POB 2390, Jerusalem, Israel.

$$
\begin{gathered}
\text { Copyright } 1976 \text { Pacific Journal of Mathematics } \\
\text { All Rights Reserved }
\end{gathered}
$$




\section{Pacific Journal of Mathematics}

Vol. 62, No. 2

February, 1976

Allan Russell Adler and Catarina Isabel Kiefe, Pseudofinite fields, procyclic

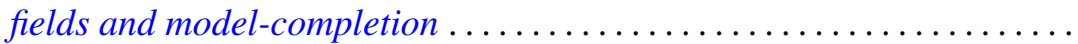

Christopher Allday, The stratification of compact connected Lie group

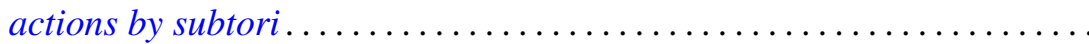

Martin Bartelt, Commutants of multipliers and translation operators .......

Herbert Stanley Bear, Jr., Ordered Gleason parts ..................

James Robert Boone, On irreducible spaces. II .....................

James Robert Boone, On the cardinality relationships between discrete

collections and open covers ............................

L. S. Dube, On finite Hankel transformation of generalized functions .......

Michael Freedman, Uniqueness theorems for taut submanifolds . . . . . . . . .

Shmuel Friedland and Raphael Loewy, Subspaces of symmetric matrices

containing matrices with a multiple first eigenvalue .............

Theodore William Gamelin, Uniform algebras spanned by Hartogs

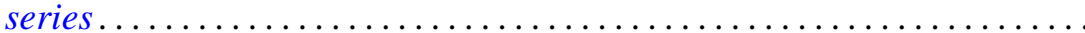

James Guyker, On partial isometries with no isometric part ............

Shigeru Hasegawa and Ryōtarō Satō, A general ratio ergodic theorem for

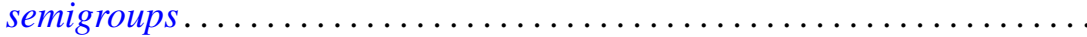

Nigel Kalton and G. V. Wood, Homomorphisms of group algebras with norm less than $\sqrt{2}$.

Thomas Laffey, On the structure of algebraic algebras...

Will Y. K. Lee, On a correctness class of the Bessel type differential operator $S_{\mu}$

Robert D. Little, Complex vector fields and divisible Chern classes ....

Kenneth Louden, Maximal quotient rings of ring extensions . .

Dieter Lutz, Scalar spectral operators, ordered $l^{\rho}$-direct sums, and the

counterexample of Kakutani-McCarthy . .

Ralph Tyrrell Rockafellar and Roger Jean-Baptiste Robert Wets, Stochastic

convex programming: singular multipliers and extended duality

singular multipliers and duality.

Edward Barry Saff and Richard Steven Varga, Geometric overconvergence of rational functions in unbounded domains ..........

Joel Linn Schiff, Isomorphisms between harmonic and P-harmonic Hardy

spaces on Riemann surfaces.

Virinda Mohan Sehgal and S. P. Singh, On a fixed point theorem of

Krasnoselskii for locally convex spaces.

Lewis Shilane, Filtered spaces admitting spectral sequence operations

Michel Smith, Generating large indecomposable continua . 